\title{
Controlling radical formation in the photoactive yellow protein chromophore
}

\author{
Ciarán R. S. Mooney, Michael A. Parkes, Andreas Iskra*and Helen H. Fielding ${ }^{\dagger}$ \\ Department of Chemistry, University College London, 20 Gordon Street, London \\ WC1H 0AJ, UK
}

To understand how photoactive proteins function, it is necessary to understand the photoresponse of the chromophore. Photoactive yellow protein $(P Y P)$ is a prototypical signalling protein. Blue light triggers trans-cis isomerisation of the chromophore covalently bound within PYP as the first step in a photocycle that results in the host bacterium moving away from potentially harmful light. At higher energies, photoabsorption has the potential to create radicals and free electrons; however, this process is largely unexplored. Here, we use photoelectron spectroscopy and quantum chemistry calculations to show that the molecular structure and conformation of the isolated PYP chromophore can be exploited to control the competition between trans-cis isomerisation and radical formation. We also find evidence to suggest that one of the roles of the protein is to impede radical formation in PYP by preventing torsional motion in the electronic ground state of the chromophore.

In nature, light drives many important processes such as photosynthesis, vision and phototaxis. At the heart of all these processes is a small chromophore whose photochemical response initiaties large-scale conformational changes in the protein wrapped around it, which in turn leads to a response at the cellular level. In addition to the desired photochemical reaction pathway, molecular chromophores are subject to competing processes such as internal conversion (IC), intersystem crossing and intramolecular vibrational redistribution and, in photoactive proteins in which the chromophore exists in a deprotonated anionic form, electron emission. ${ }^{[1+4]}$ The role of the protein in controlling the competition between these process is still not understood fully.

PYP is the primary photoreceptor for the negative photoactic response of the Halorhodospira

\footnotetext{
*Present address: Physical and Theoretical Chemistry Laboratory, University of Oxford, South Parks Road, Oxford OX1 3QZ, UK

${ }^{\dagger}$ Corresponding author: h.h.fielding@ucl.ac.uk
} 
halophila bacterium to the potentially harmful effects of blue light and has been the subject of numerous experimental and theoretical studies as a model photoreceptor. ${ }^{[5]}\left[{ }^{[}\right]$The chromophore of PYP is derived from trans para-coumaric acid $(p C A)$ and exists in its deprotonated phenoxide form $\left(p \mathrm{CA}^{-}\right)$in the ground electronic state (Fig. 1). Numerous gas-phase studies of isolated model PYP chromophores have shown that the protein environment does not influence the absorption spectrum significantly but, following excitation around $400 \mathrm{~nm}$, it plays a key role in directing trans-cis isomerisation of the chromophore and impeding electron emission and radical formation. ${ }^{[9-18]}$ Electron emission has also been observed following excitation at shorter wavelengths in PYP [12] and in isolated PYP chromophores in solution, ${ }^{[19]}$ although the photoresponse of the isolated chromophore in vacuo at short wavelengths is largely unexplored.

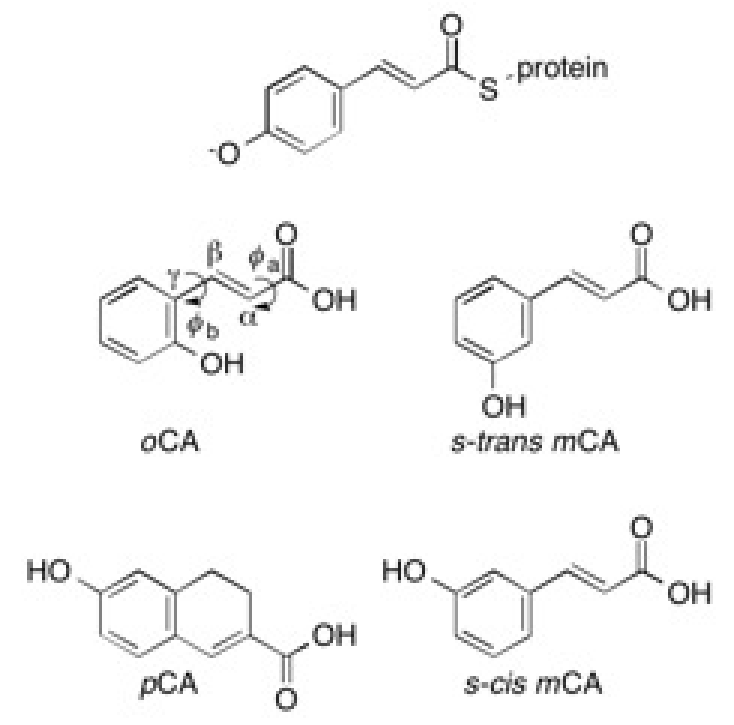

Figure 1: Structures of the PYP chromophore in the protein (top) and those employed in this work. The conformation of $o \mathrm{CA}$ around the bond linking the $\beta$ and $\gamma$ atoms is well-defined, but for $m \mathrm{CA}$ it is undefined and exists in both s-cis and s-trans conformations, where s-cis and s-trans define the position of the phenol $\mathrm{OH}$ group with respect to the $\beta$ hydrogen. As drawn, $\phi_{a}=0^{\circ}$.

Here, we use anion photoelectron spectroscopy ${ }^{[20-22]}$ and quantum chemistry calculations to investigate the effect of molecular structure on the competition between IC and electron emission following $364-315 \mathrm{~nm}$ excitation of deprotonated anions derived from $p$ CA and two of its isomers, trans ortho-coumaric acid (oCA) and trans meta-coumaric acid (mCA) (Fig. 1). Photoelectron spectra of $o \mathrm{CA}^{-}, m \mathrm{CA}^{-}$and $p \mathrm{CA}^{-}$are recorded as a function of electron kinetic energy (eKE) and presented in Fig. 2 2 as a function of electron binding energy, eBE $=h v-\mathrm{eKE}$. All the spectra have similar profiles: a broad feature at low eBE and a sharp feature at high eBE.

The maxima of the broad features remain at constant eBE for all photon energies, signifying a direct photodetachment (PD) process. The maxima of these features are close to the vertical 

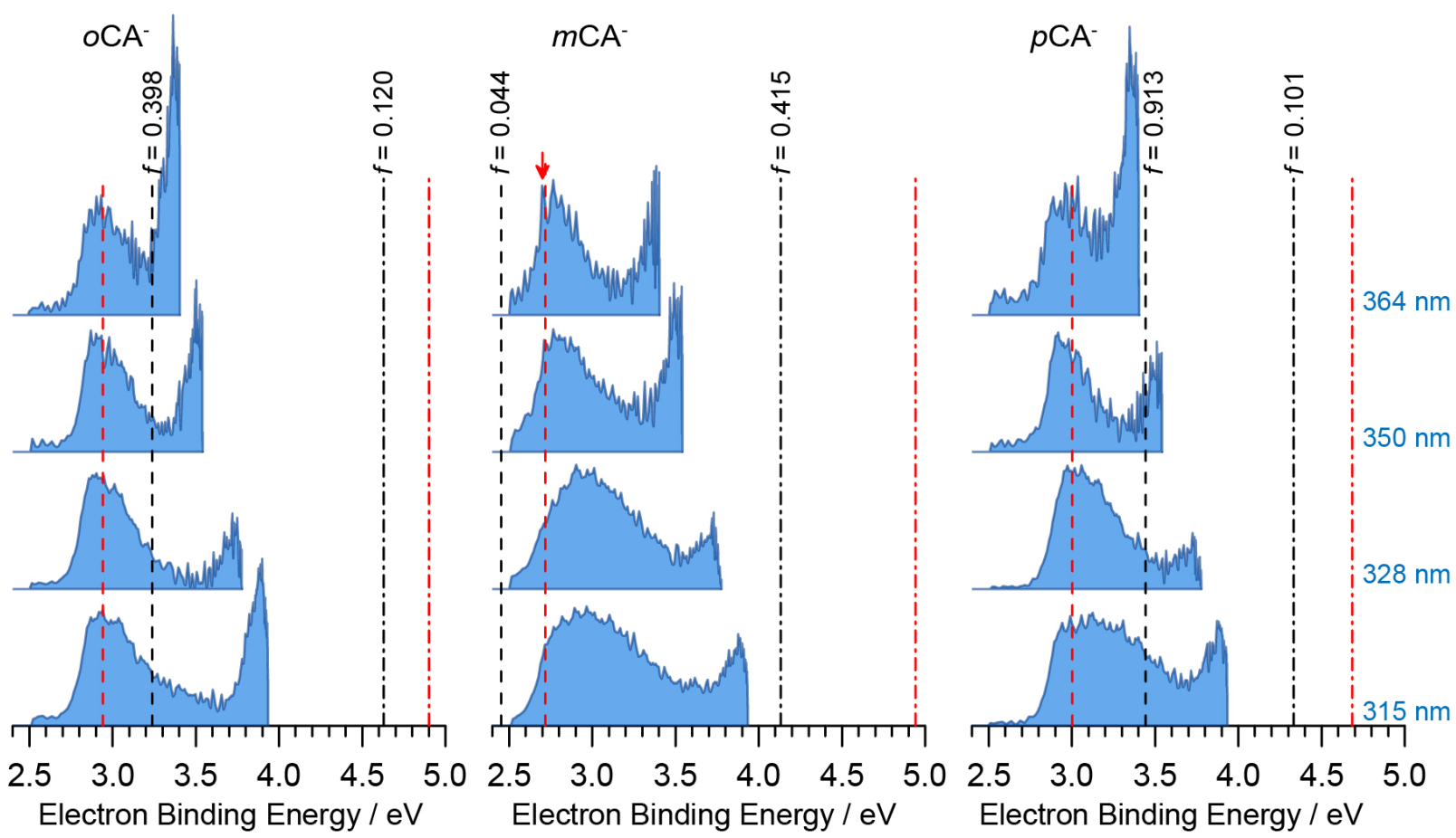

Figure 2: Photoelectron spectra of $o \mathrm{CA}^{-}, m \mathrm{CA}^{-}$and $p \mathrm{CA}^{-}$recorded at $364 \mathrm{~nm}(3.41 \mathrm{eV}), 350 \mathrm{~nm}$ $(3.54 \mathrm{eV}), 328 \mathrm{~nm}(3.78 \mathrm{eV})$ and $315 \mathrm{~nm}(3.94 \mathrm{eV})$, plotted as a function of eBE. Spectra have been normalised to the maxima of the low eBE features. Vertical red lines mark the VDEs calculated for the lowest energy (planar) configurations of the chromophores in their phenolate (dashed) and carboxylate (dot-dashed) forms. The vertical red arrow marks the position of the peak at $2.70 \pm 0.05 \mathrm{eV}$ in the $364 \mathrm{~nm}$ spectrum of $\mathrm{mCA}^{-}$(see text). Vertical black lines mark the calculated VEEs (with oscillator strengths, $f$ ) of the $1 \pi \pi^{*}$ (dashed) and $2 \pi \pi^{*}$ (dot-dashed) states of the phenolate forms of the anions in their lowest energy (planar) configurations.

detachment energies (VDEs) we calculated for the phenolate forms of the chromophores $(2.7$ $3.0 \mathrm{eV}$ ), which are $\sim 2 \mathrm{eV}$ lower than those calculated for the carboxylate forms $(4.7-4.9 \mathrm{eV})$ (Fig. 2). Thus, our measurements support earlier suggestions that the coumaric acid chromophores are formed in their phenolate forms during the electrospray-ionisation process. [13]17]

For $m \mathrm{CA}^{-}$and $p \mathrm{CA}^{-}$, the broad features change shape on their high eBE side as the photon energy is increased. This can be explained in terms of resonant excitation of the $2^{1} \pi \pi^{*}$ states (calculated VEEs around 4.2 and $4.3 \mathrm{eV}$ for $m \mathrm{CA}^{-}$and $p \mathrm{CA}^{-}$, respectively) followed by autodetachment, i.e. indirect PD (Fig. 3). A similar effect was observed in photoelectron spectra of the deprotonated green fluorescent protein (GFP) chromophore anion following resonant excitation of the $2^{1} \pi \pi^{*}$ state. ${ }^{[22]}$ For $o \mathrm{CA}^{-}$, the broad feature does not change shape, which is consistent with the $2^{1} \pi \pi^{*}$ state lying higher in energy (calculated VEE $\sim 4.6 \mathrm{eV}$ ) and suggests that it is inaccessible at these photon energies.

The sharp features shift to higher eBE with increasing photon energy, signifiying an indirect 


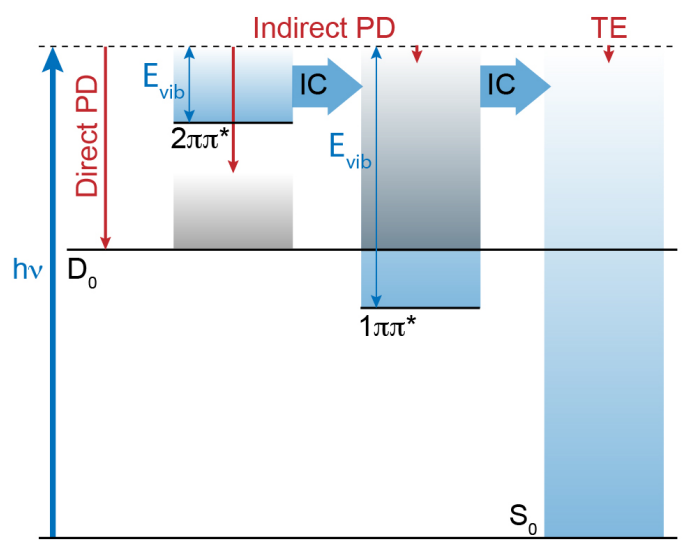

Figure 3: Schematic diagram illustrating electron emission processes (red arrows) following photoexcitation $(h v)$. Direct photodetachment (PD) gives photoelectrons with maximum eKE around $h v-E\left(\mathrm{D}_{0}-\mathrm{S}_{0}\right)$. Resonant excitation of the $2^{1} \pi \pi^{*}$ state with excess vibrational energy $E_{\mathrm{vib}}=h v-E\left(2^{1} \pi \pi^{*}-\mathrm{S}_{0}\right)$ (blue shading) may be followed by indirect PD generating electrons with eKE around $E_{\mathrm{PD}}=E\left(2^{1} \pi \pi^{*}-\mathrm{D}_{0}\right)$, as a result of the propensity for conserving vibrational energy during the detachment process (grey shading), or by IC to $1^{1} \pi \pi^{*}$, followed by indirect $\mathrm{PD}$ or IC to $\mathrm{S}_{0}$. Subsequent statistical TE from $\mathrm{S}_{0}$ is characterised by electrons with eKE $\sim 0 \mathrm{eV}$.

electron emission process. PD from $1^{1} \pi \pi^{*}$ and thermionic emission (TE) from the vibrationally hot ground electronic state of the anion are possible electron emission pathways (Fig. 3). The exponential profile is characteristic of TE. Similar features have been observed in photoelectron spectra of other molecular anions, such as the $p$-benzoquinone radical anion, 23$]$ and deprotonated GFP chromophore anion, $[24]$ and, moreover, TE from a hot ground state is consistent with IC back to the ground electronic state, which has been observed to occur on a 52 ps timescale in methyl-terminated (ether) $p \mathrm{CA}^{-}$following photoexcitation of $\mathrm{S}_{1}$ at $3.1 \mathrm{eV}$. [10]

The vertical excitation energies (VEEs) of the $1^{1} \pi \pi^{*}$ states in $o \mathrm{CA}^{-}$and $p \mathrm{CA}^{-}$have large oscillator strengths and are close to the lowest photon energy (Fig. 2). Thus, the $3.41 \mathrm{eV}$ photoelectron spectra of $o \mathrm{CA}^{-}$and $p \mathrm{CA}^{-}$are dominated by low eKE photoelectrons arising from indirect PD from the $1^{1} \pi \pi^{*}$ state and TE from $S_{0}$ (Fig. 3). In contrast, the VEE of the $1^{1} \pi \pi^{*}$ state in $m \mathrm{CA}^{-}$is much lower in energy and weaker and, consequently, the $3.41 \mathrm{eV}$ photoelectron spectrum of $m \mathrm{CA}^{-}$is dominated by direct PD (Fig. 3).

The $\mathrm{S}_{0} \rightarrow 1^{1} \pi \pi^{*}$ transitions are all dominated by transitions from the highest occupied molecular orbitals (HOMOs) (Fig. 4). For $o \mathrm{CA}^{-}$and $p \mathrm{CA}^{-}$, the $\mathrm{HOMOs}$ are delocalised across the whole molecule with $\pi$ bonding character across the $\mathrm{C}=\mathrm{C}$ double bond between the phenoxide and carboxylic acid groups, whereas for $m \mathrm{CA}^{-}$the $\mathrm{HOMO}$ is a pair of out-of-phase $\pi$ orbitals on the phenoxide moiety and there is negligible electron density on the propenoic acid moiety. The $\mathrm{S}_{0} \rightarrow 1^{1} \pi \pi^{*}$ transitions in $o \mathrm{CA}^{-}$and $p \mathrm{CA}^{-}$are dominated by $\pi \rightarrow \pi^{*}$ 

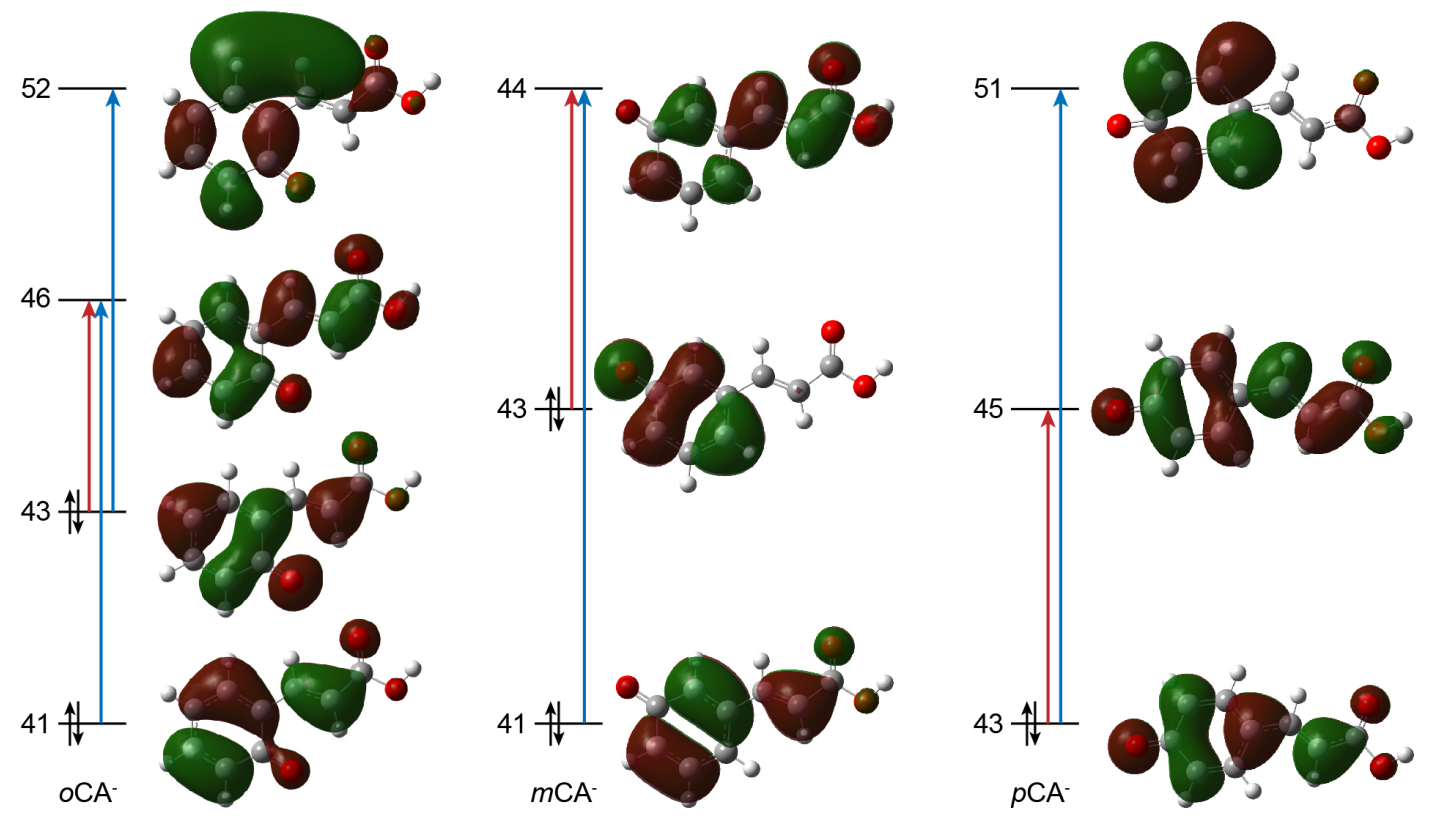

Figure 4: Main ground-state molecular orbitals involved in the $1^{1} \pi \pi^{*}$ and $2^{1} \pi \pi^{*}$ transitions for $o \mathrm{CA}^{-}, s-c i s m \mathrm{CA}^{-}$and $p \mathrm{CA}^{-}$.

transitions on the $\mathrm{C}=\mathrm{C}$ double bond and the $\mathrm{S}_{0} \rightarrow 1^{1} \pi \pi^{*}$ transition in $m \mathrm{CA}^{-}$involves $\pi \rightarrow \pi^{*}$ transitions on the phenoxide group and charge transfer to the propenoic acid moiety. Thus, moving the position of the $\mathrm{O}^{-}$group on the phenoxide moiety provides a means of controlling the conjugation, which in turn controls the competition between resonant excitation of the $1^{1} \pi \pi^{*}$ state and direct detachment.

Since the $m \mathrm{CA}^{-}$photoelectron spectrum is dominated by direct PD, it can be modelled by calculating the Franck-Condon overlap between the ground electronic states of the anion and the neutral radical (Fig. 5). The excellent agreement between the calculated and experimental spectra allows us to assign the well-resolved feature in the experimental photoelectron spectrum at $2.70 \pm 0.05 \mathrm{eV}$ to the $0-0$ transition $(2.71 \mathrm{eV})$ and tells us that the adiabatic detachment energy is equivalent to the VDE. We also determine that the photoelectron spectrum is dominated by a progression in the $v_{11}$ mode (harmonic frequency $531 \mathrm{~cm}^{-1}$ ), corresponding to an in-plane vibration (Fig. 5).

Curiously, the $o \mathrm{CA}^{-}$and $p \mathrm{CA}^{-}$photoelectron specta reveal significant photoemission at lower eBEs than the calculated VDEs (Fig. 2). Rotation around the single bond between the $\mathrm{C}=\mathrm{C}$ double bond and the phenoxide group has been shown to facilitate trans-cis isomerisation in PYP. ${ }^{925]}$ In Fig. 6 the $3.41 \mathrm{eV}(364 \mathrm{~nm})$ experimental photoelectron spectrum for $p \mathrm{CA}^{-}$is shown together with the positions of the calculated VDEs for the deprotonated chromophore anion in its optimised planar geometry and geometries optimised with $\phi_{a}=90^{\circ}$ or $\phi_{b}=90^{\circ}$. 


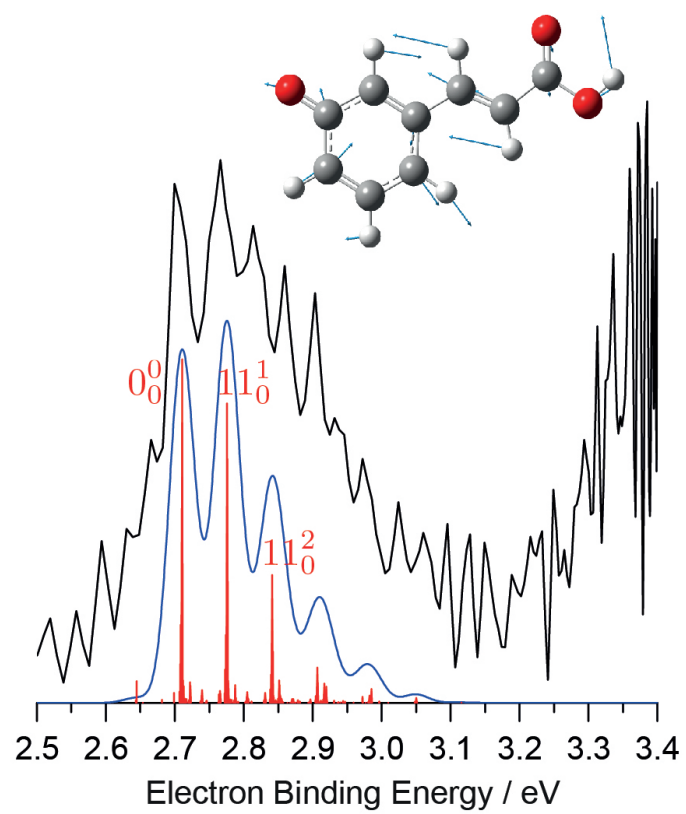

Figure 5: Calculated photoelectron stick spectrum of the phenolate form of the deprotonated $s$-cis $m \mathrm{CA}^{-}$chromophore at $T=300 \mathrm{~K}$ (red) and stick spectrum convoluted with Gaussian instrument profiles with $\mathrm{HWHM}=18 \mathrm{meV}$ (blue) compared with the experimental $364 \mathrm{~nm}$ $(3.41 \mathrm{eV})$ photoelectron spectrum (black). Inset: Atomic displacement vectors of the dominant $v_{11}$ normal mode of the neutral radical.

The substantial photoelectron signal lying below the VDE for the planar chromophore lies above the VDEs for the $90^{\circ}$ twisted chromophores and therefore we attribute the photoelectron signal below the VDE for the planar chromophore to PD from twisted chromophore anions. This suggests that one of the reasons the protein environment in PYP restricts torsional motion in the ground electronic state of the anion is to raise the threshold for direct PD and thus impede electron emission and radical formation. Hydrogen-bonding between the $\mathrm{O}^{-}$on the phenoxide group and the $\alpha$ hydrogen in $o \mathrm{CA}^{-}$is likely to restrict rotation around $\phi_{b}$, perhaps explaining why the photoemission at lower eBEs is less significant than for $p \mathrm{CA}^{-}$.

At the highest photon energy $(3.94 \mathrm{eV})$, the contribution from low eKE electrons increases, most noticeably for $o \mathrm{CA}^{-}$. This could be attributed to detachment to higher lying continua or access to a new conical intersection providing an efficient radiationless decay path back to $\mathrm{S}_{0}$. Unravelling the decay pathway will require femtosecond time-resolved photoelectron spectroscopy measurements, $26+28]$ which are currently underway in our laboratory.

In summary, we have studied the competition between electron emission and internal conversion in the model PYP chromophore, $p \mathrm{CA}^{-}$, and two of its isomers. We show that moving the position of the $\mathrm{O}^{-}$group on the phenoxide moiety of the chromophore changes the branching 


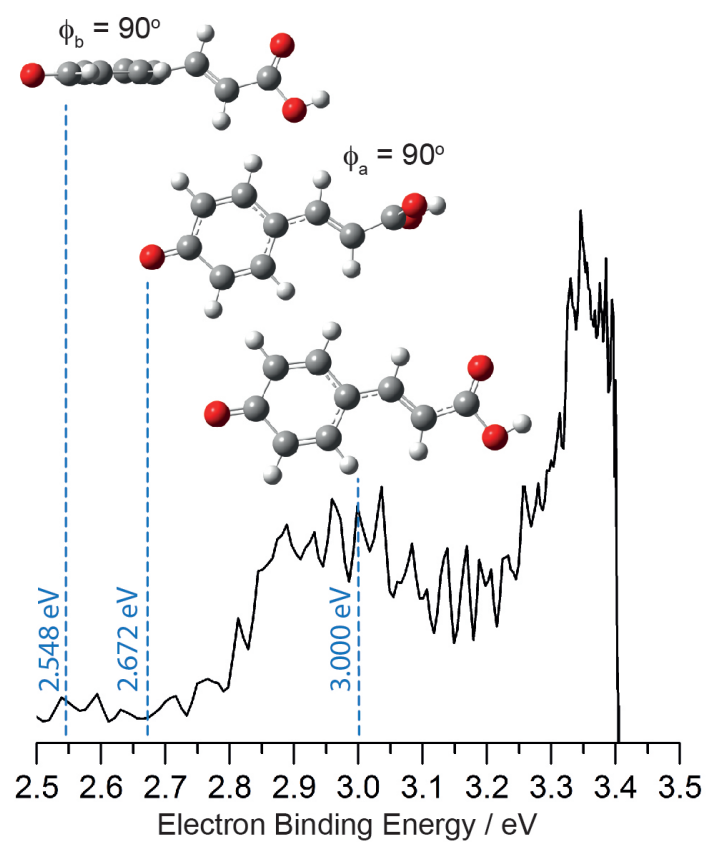

Figure 6: $3.41 \mathrm{eV}(364 \mathrm{~nm})$ photoelectron spectrum of the phenolate form of the deprotonated $p \mathrm{CA}^{-}$chromophore shown with calculated VDEs for the minimum energy geometry and geometries optimised with $\phi_{a}=90^{\circ}$ and $\phi_{b}=90^{\circ}$.

ratio between direct PD and resonant excitation of $1 \pi \pi^{*}$ and $2 \pi \pi^{*}$ states, which subsequently undergo IC to lower electronic states followed by autodetachment or TE. We also show that torsional motions in the ground electronic state are responsible for lowering the VDE, suggesting that one of the reasons the protein maintains the planarity of the chromophore in its ground electronic state is to impede electron emission and radical formation. These results illustrate how modifications to the chemical structure away from the excited state reaction coordinate can control the competition between dynamics on the excited state and radical formation and demonstrate the importance of low-frequency single bond rotations.

\section{Acknowledgement}

This work was supported by EPSRC grants EP/L005646/1 and EP/D054508/1. We acknowledge use of the EPSRC UK National Service for Computational Chemistry Software (NSCCS) at Imperial College London and discussions with Alexandra Simperler, and the iOpenShell Center for Computational Studies of Electronic Structure and Spectroscopy of Open-Shell and Electronically Excited Species, supported by the National Science Foundation. We are grateful to Christopher West and Jan Verlet (Durham) who enabled us to record preliminary data using their anion photoelectron spectrometer. 


\section{References}

[1] D. S. Larsen, I. H. M. van Stokkum, M. Vengris, M. A. van der Horst, F. L. de Weerd, K. J. Hellingwerf, R. van Grondelle, Biophys. J. 2004, 87, 1858-1872.

[2] J. Zhu, L. Paparelli, M. Hospes, J. Arents, J. T. M. Kennis, I. H. M. Van Stokkum, K. J. Hellingwerf, M. L. Groot, J. Phys. Chem. B 2013, 117, 11042-11048.

[3] A. M. Bogdanov, A. S. Mishin, I. V. Yampolsky, V. V. Belousov, D. M. Chudakov, F. V. Subach, V. V. Verkhusha, S. Lukyanov, K. A. Lukyanov, Nat. Chem. Biol. 2009, 5, 459-61.

[4] K. M. Solntsev, D. Ghosh, A. Amador, M. Josowicz, A. I. Krylov, J. Phys. Chem. Lett. 2011, 2, 2593-2597.

[5] T. E. Meyer, Biochim. Biophys. Acta 1985, 806, 175-183.

[6] W. W. Sprenger, W. D. Hoff, J. P. Armitage, K. J. Hellingwerf, J. Bacteriol. 1993, 175, 30963104.

[7] K. J. Hellingwerf, J. Hendriks, T. Gensch, J. Phys. Chem. A 2003, 107, 1082-1094.

[8] F. Schotte, H. Sun Cho, V. R. I. Kaila, H. Kamikubo, N. Dashdorj, E. R. Henry, T. J. Graber, R. Henning, M. Wulff, G. Hummer, M. Kataoka, P. A. Anfinrud, Proc. Natl. Acad. Sci. 2012, 109, 19256-19261.

[9] G. Groenhof, M. Bouxin-Cademartory, B. Hess, S. P. de Visser, H. J. C. Berendsen, M. Olivucci, A. E. Mark, M. A. Robb, J. Am. Chem. Soc. 2004, 126, 4228-4233.

[10] I-R. Lee, W. Lee, A. H. Zewail, Proc. Natl. Acad. Sci. U. S. A. 2006, 103, 258-262.

[11] L. Lammich, J. Rajput, L. H. Andersen, Phys. Rev. E 2008, 78, 051916.

[12] M. de Groot, E. V. Gromov, H. Köppel, W. J. Buma, J. Phys. Chem. B 2008, 112, 4427-4434.

[13] T. Rocha-Rinza, O. Christiansen, J. Rajput, A. Gopalan, D. B. Rahbek, L. H. Andersen, A. V. Bochenkova, A. A. Granovsky, K. B. Bravaya, A. V. Nemukhin, K. L. Christiansen, M. Brøndsted Nielsen, J. Phys. Chem. A 2009, 113, 9442-9449.

[14] T. Rocha-Rinza, O. Christiansen, D. B. Rahbek, B. Klærke, L. H. Andersen, K. Lincke, M. Brøndsted Nielsen, Chem. Eur. J. 2010, 16, 11977-11984. 
[15] S. Smolarek, A. Vdovin, D. L. Perrier, J. P. Smit, M. Drabbels, W. J. Buma, J. Am. Chem. Soc. 2010, 132, 6315-6317.

[16] S. Smolarek, A. Vdovin, E. M. M. Tan, M. de Groot, W. J. Buma, Phys. Chem. Chem. Phys. 2011, 13, 4393-4399.

[17] D. Zuev, K. B. Bravaya, T. D. Crawford, R. Lindh, A. I. Krylov, J. Chem. Phys. 2011, 134, 034310.

[18] M. Uppsten, B. Durbeej, J. Comput. Chem. 2012, 33, 1892-1901.

[19] D. S. Larsen, M. Vengris, I. H. M. van Stokkum, M. a. van der Horst, F. L. de Weerd, K. J. Hellingwerf, R. van Grondelle, Biophys. J. 2004, 86, 2538-50.

[20] A. R. McKay, M. E. Sanz, C. R. S. Mooney, R. S. Minns, E. M. Gill, H. H. Fielding, Rev. Sci. Instrum. 2010, 81, 123101.

[21] C. R. S. Mooney, M. E. Sanz, A. R. McKay, R. J. Fitzmaurice, A. E. Aliev, S. Caddick, H. H. Fielding, J. Phys. Chem. A 2012, 116, 7943-7949.

[22] C. R. S. Mooney, M. A. Parkes, L. Zhang, H. C. Hailes, A. Simperler, M. J. Bearpark, H. H. Fielding, J. Chem. Phys. 2014, 140, 205103.

[23] D. A. Horke, Q. Li, L. Blancafort, J. R. R. Verlet, Nat. Chem. 2013, 5, 711-7.

[24] C. W. West, A. S. Hudson, S. L. Cobb, J. R. R. Verlet, J. Chem. Phys. 2013, 139, 071104.

[25] A. D. Stahl, M. Hospes, K. Singhal, I. van Stokkum, R. van Grondelle, M. L. Groot, K. J. Hellingwerf, Biophys. J. 2011, 101, 1184-1192.

[26] R. S. Minns, D. S. N. Parker, T. J. Penfold, G. A. Worth, H. H. Fielding, PCCP 2010, 12, 15607-15615.

[27] R. Spesyvtsev, O. M. Kirkby, M. Vacher, H. H. Fielding, PCCP 2012, 14, 9942-9947.

[28] C. R. S. Mooney, D. A. Horke, A. S. Chatterley, A. Simperler, H. H. Fielding, J. R. R. Verlet, Chem. Sci. 2013, 4, 921. 


\section{Keywords}

biophysics, chromophores, photochemistry, proteins, radical formation

\section{Graphical table of contents}

The role of chemical structure and low frequency bond rotations on the control of the competition between isomerisation and electron emission (radical formation) in the chromophore of photoactive yellow protein.

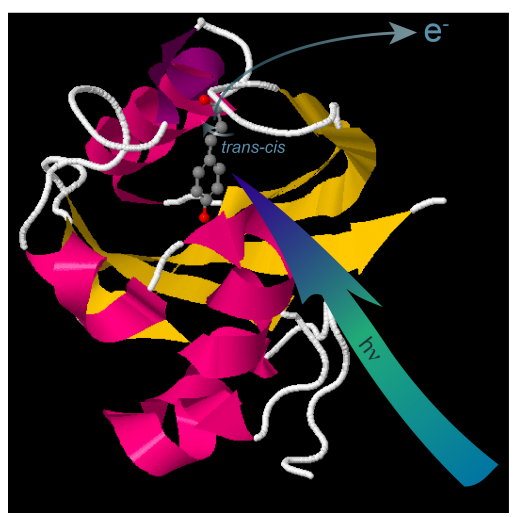

